\title{
Severe diphtheria in a partially immunised UK 4 year old
}

H Dale1, F Shackley1, C Waruiru1 B. Charles ${ }^{1}$ S. Thompson'1, M Chand 2 G Amirthalingam 21 Sheffield Children's Hospital NHS Foundation Trust; 2 PHE Colindale

\section{Background}

Diphtheria is a serious and life-threatening infection. Between 2010 - 2016 there were 12 reported cases of toxigenic diphtheria in the $\mathrm{UK}^{1}$, and 35 confirmed cases across Europe in 2014; approximately $1 / 3$ rd of these were children $<15 \mathrm{yrs}^{2}$. Since 2015, two

unvaccinated children have died of diphtheria in Europe ${ }^{3}$, partly relating to delay in administration of anti-toxin due to global shortages. Due to the rarity of this infection in the UK, delay in diagnosis and recognising complications, can lead to delay in effective patient care. Severe disease can also occur in partially immunised children.

\section{Case Presentation}

A 4 year old Slovak boy, presented with a croup-like illness. Initial respiratory secretions isolated adenovirus, Moraxella and Haemophilus. He developed severe upper airway obstruction requiring ventilation for 5 days. Extubation was difficult due to persistent stridor, and he required a tracheostomy. On second attempted extubation on day 5 , an unusual grey pharyngeal membrane was seen raising the suspicion of diphtheria; despite having been partially immunised at 2 and 12 months.

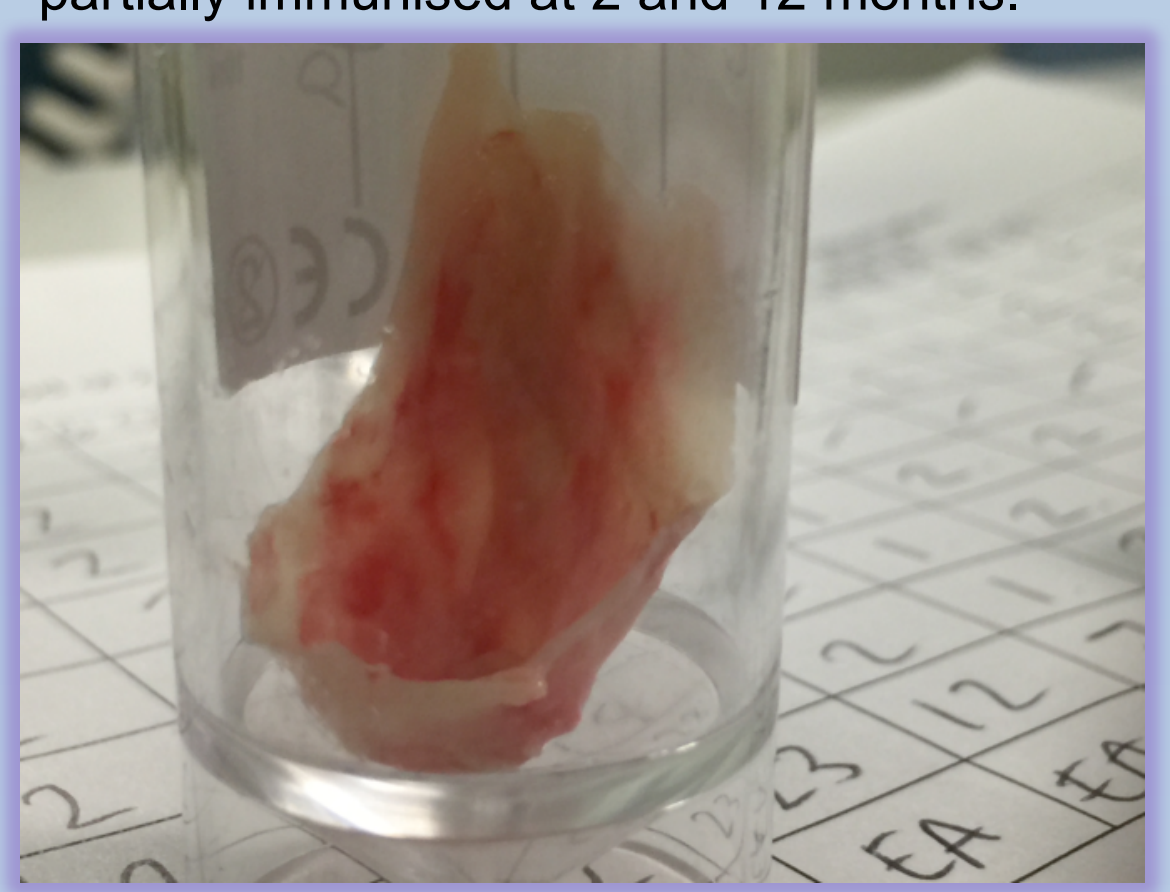

Fig. 1. Grey pharyngeal membrane found on extubation

\section{Diphtheria Facts}

Corynebacterium diphtheriae (C.ulcerans if toxin producing)

Droplet transmission in close contact Exotoxin $=$ toxicity in $8.1 \%$ leading to systemic involvement; myocarditis, neuropathy, renal failure and death

- Cutaneous or respiratory infection

Vaccinated people can be asymptomatic carriers

\section{Initial management}

He was commenced on erythryomycin and penicillin for diphtheria, cefuroxime for Haemophilus, and notified to public health. He was treated with an initial dose of 40,000 units of diphtheria anti-toxin (DAT) in line with national guidance for pharyngeal and laryngeal disease ${ }^{4}$. Toxigenic $C$. diptheriae was confirmed by PCR with Elek positivity (a measure of toxicity), and resistance to penicillin. He was switched to clarithromycin and ciprofloxacin.

\section{Clinical progress}

He deteriorated further after 5 days with respiratory compromise, pulmonary haemorrhage and evidence of myocarditis. He had raised troponin. His ECG showed left ventricular hypertrophy, ST segment ischaemic changes and prolonged QT. He received a further 60,000 units of DAT (total 100,000 units) with no adverse reaction. Ciprofloxacin was changed to vancomycin due to prolonged QT.

\section{Further Complications}

He developed renal impairment and hyponatraemia; renal USS showed echobright kidneys.

He developed persistent fever 2 days after DAT with no other features of serum sickness and had an extensive infection screen; CSF WCC $<1$ but raised protein, and neuroimaging was normal A repeat echocardiogram showed coronary artery dilation. National experts
- advised that while Kawasaki-like features were present, it was likely all diphtheria related and there was no indication for immunoglobulin.

Aspirin was commenced at anti-platelet dose. His fever settled spontaneously.

- He exhibited a bisphasic diphtheritic polyneuropathy with global weakness,

hypotonia and hyporeflexia. This was insidious at onset, masked by sedation and presumed to be due to critical illness neuropathy and prolonged use of steroids for upper airway obstruction. Following a period of stability at week 6 , he returned to PICU with respiratory failure requiring ventilation. He was found to have progressive neuropathy; bulbar, diaphragmatic (diagnosed on USS) and lateral rectus palsies, with left ptosis. Treatment with steroids and immunoglobulins for a GuillainBarre like illness were considered due to raised CSF protein and EMG studies. However, as all features were consistent with diphtheritic neuropathy ${ }^{5}$, and immunoglobulins and steroids could have adverse effects, supportive treatment was advised.

\section{Outcome}

Despite his significant multisystem disease and prolonged and severe neurological compromise, his tracheostomy was reversed after 2 months and he was discharged after 3 months. On long term follow up coronary artery size had regressed with no aneurysm formation.

\section{Discussion}

- UK guidelines ${ }^{4}$ recommend IV or IM administration of DAT. However, in this case it was given subcutaneously after much consideration, due to an unstable airway and risk of anaphylaxis with IM/ IV administration, in addition the DAT obtained was for SC use only.

Coronary artery dilatation has not been documented in diphtheritic carditis. However, it may not have been investigated in other cases and true incidence should be further explored.

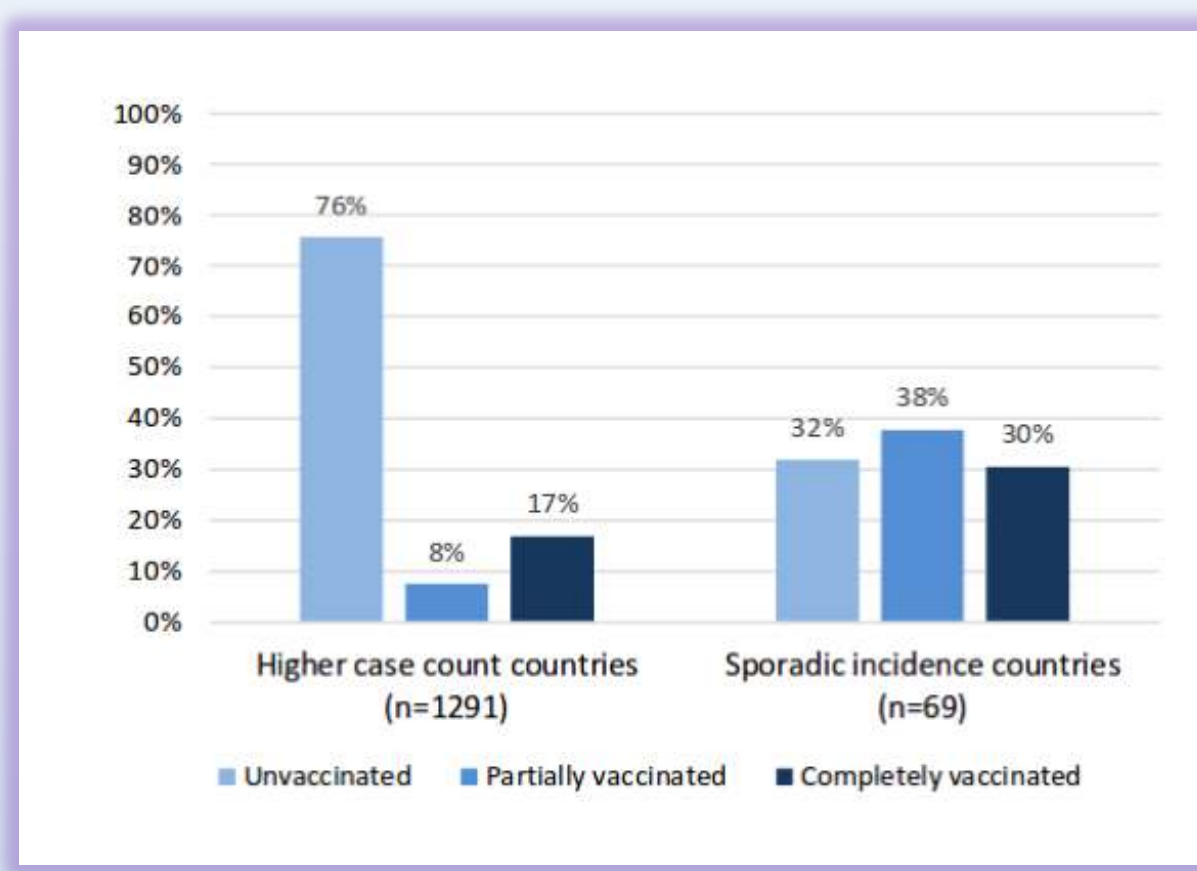

Fig 2. Vaccination status of cases in high case count countries versus sporadic incidence countries $^{6}$

- The persistent fever and thrombophilia led us to consider a Kawasaki-like illness, however, was felt to be part of diphtheritic carditis and immunoglobulins were not indicated.

- Lack of familiarity with the prolonged clinical picture of diphtheria led to some delay in recognition of his neurological symptoms

- Infections in vaccinated and partially vaccinated people, in sporadic incidence countries (where age of cases tends to be higher, and primary vaccination coverage rates are higher) may relate to waning immunity (see fig. 2)

- Timely administration of DAT can reduce risk of neurological sequelae; delay in diagnosis and global shortages of DAT make this challenging

\section{Learning points}

- Severe diphtheria may occur in partially immunised children in low incidence countries.

- Neurological involvement may be insidious and diagnosis may be delayed in countries with little experience.

- Diphtheria associated coronary artery dilatation is not well documented, and should be further explored.

- Diphtheria anti-toxin can be safely given by subcutaneous infusion; this may be useful to document in future national guidelines.

References: 1) Diphtheria notifications and deaths: England and Wales 1986 -2014. Public Health England. March 2014,2) European Centre for Disease Prevention and Control. Annual

Epidemiological Report 2016 - Diphtheria. Stockholm: ECDC; 2016 3) Oxford Vaccine Group. Diphtheria: http://vk.ovg.ox.ac.uk/diphtheria 4) PHE immunoglobulin handbook 2017 6) Diphtheritic

Polyneuropathy Clinical Analysis of Severe Forms. Piridov et al, JAMA, 2001 6) Review of the Epidemiology of Diphtheria - 2000- 2016. WHO 


\section{Background}

Diphtheria is a serious and life-threatening infection, Between 2010 - 2016 there were 12 reported cases of toxigenic diptheria in the $\mathrm{UK}^{1}$, and 35 confirmed cases across Europe in 2014; approximately $1 / 3 \mathrm{rd}$ of these were children $<15 \mathrm{yrs}^{2}$. Since 2015, two unvaccinated children have died of diphtheria in Europe ${ }^{3}$, partly relating to delay in administration of anti-toxin due to global shortages. Due to the rarity of this infection in the UK, delay in diagnosis and recognising complications, can lead to delay in effective patient care. Severe disease can also occur in partially immunised children.

\section{Case Presentation}

A 4 year old partially immunised Slovak boy, presented with a croup-like illness. Initial respiratory secretions isolated adenovirus Moraxella and Haemophilus. He developed severe upper airway obstruction requiring ventilation for 5 days. Extubation was difficult due to persistent stridor, and he had a tracheostomy. On second attempted extubation an unusual grey pharyngeal membrane was seen raising the suspicion of diptheria.

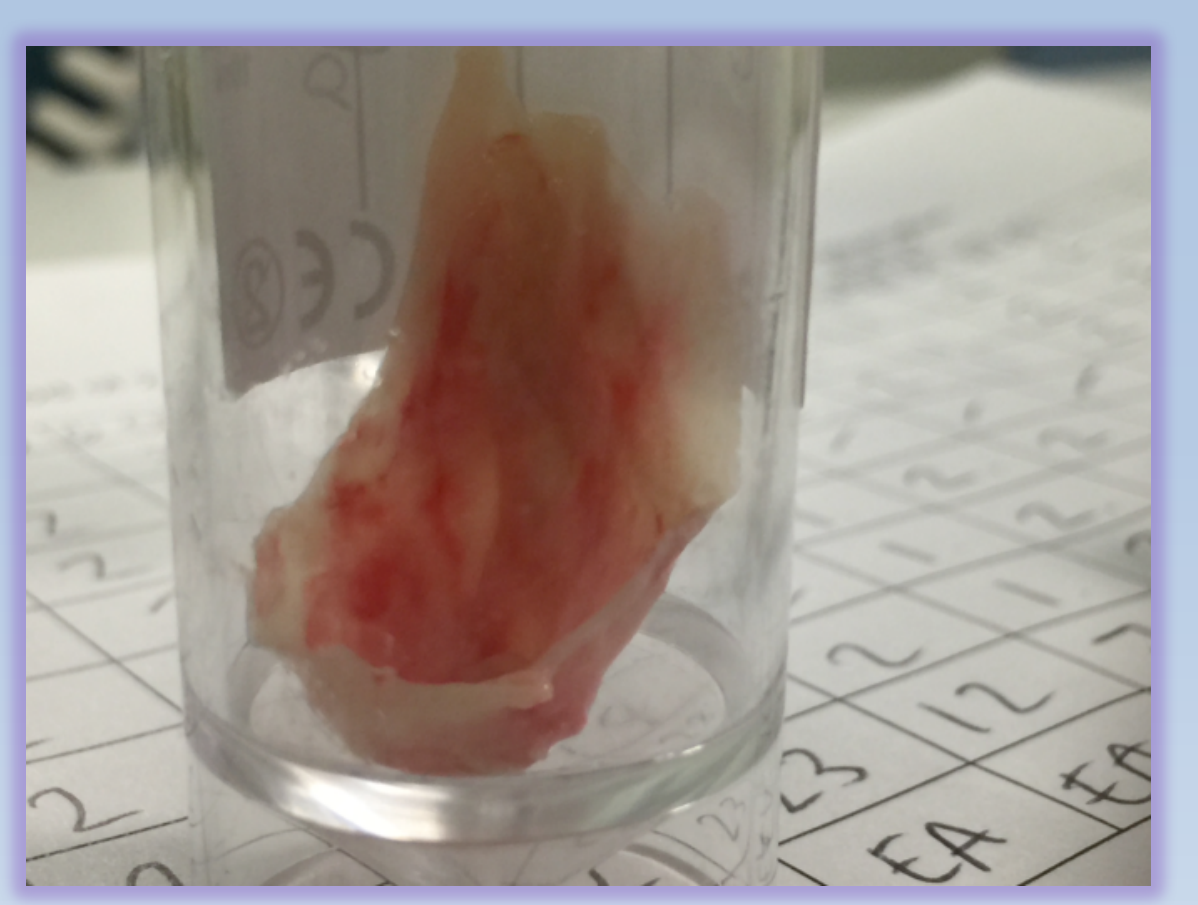

Initial management

He was commenced on erythryomycin, and notified to public health. He was treated with an initial dose of 40,000 units of diptheria anti-toxin (DAT) in line with national guidance for pharyngeal and laryngeal disease ${ }^{4}$. Toxigenic C. diptheriae was confirmed by PCR with Elek positivity (a measure of toxicity), with resistance to penicillin

\section{Clinical progress}

He deteriorated further after 5 days with respiratory compromise, pulmonary haemorrhage \& evidence of myocarditis. He had raised troponin. His ECG showed left ventricular hypertrophy, ST segment ischaemic changes and prolonged QT. He received a further 60,000 units of DAT (total 100,000 units) with no adverse reaction. His antibiotics were changed to ......due to cardiac concerns.

\section{Further Complications}

He developed renal impairment and hyponatraemia; renal USS showed echobright kidneys.

- He developed persistent fever with no other features of serum sickness and had an extensive infection screen; CSF WCC $<1$ but raised protein, and neuroimaging normal. A repeat echocardiogram showed evidence of coronary artery dilation. The

\section{Diphtheria Facts}

- Corynebacterium diphtheriae (C.ulcerans if toxin producing)

- Droplet transmission in close contact

- Exotoxin = toxicity in $8.1 \%$ leading to myocarditis, neusystemic ropathy, renal failure and death

- Cutaneous or respiratory infection

- Vaccinated people can be asymptomatic carriers
- treatment options at this stage were discussed with experts nationally. IVIG which would be indicated for a kawasaki-like illness was not given to avoid the risk of immune complex activation. Aspirin was commenced at anti-platelet dose. His fever settled spontaneously.

- He also exhibited a bisphasic diphtheritic polyneuropathy with global weakness, hypotonia and hypoeflexia. This was insidious at onset, masked by sedation and presumed to be due to critical illness neuropathy and prolonged use of steroids fo upper airway obstruction. Following a period of stability at week $\mathbf{x}$, he returned to PICU with respiratory failure requiring ventilation. He was found to have progressive neuropathy; bulbar, diaphragmatic (diagnosed on USS) and lateral rectus palsies. Treatment with steroids and IVIG for a Guillain-Barre like illness wereconsidered due to raised CSF protein and EMG studies. However, as all features were consistent with diphtheritic neuropathy, and IVIG and steroids could have adverse effectis, supportive treatment was advised.

\section{Outcome}

Despite his significant multisystem disease and prolonged and severe neurological compromise his tracheostomy was reversed after 2 months and he was discharged after 3 months. On long term follow up coronary artery size has regressed with no aneurismal formation.

\section{Discussion}

UK guidelines ${ }^{4}$ recommend IV or IM administration of antitoxin. However, in this case it was given $\mathrm{SC}$ after much deliberation, due to concerns regarding this child's airway and risk of anaphylaxis with IM/ IV administration, and that the antitoxin obtained was for subcutaneous use only. Coronary artery dilatation has not been
- fever and thrombophillia led us to consider a Kawasaki like illness, and while immunoglobulin was not given the changes appear to have resolved

- Lack of familiarity with prolonged clinical picture of diptheria led to some delay in recognition of his neurological symptoms

- Caution of IVIG following antitoxin is highlighted, as there is risk of immune complex formation.

- Infection in a partially immunised child, in a country with a sporadic incidence (where age of cases tends to be higher) may relate to waning immunity (see fig. 2)

- Timely administration of DAT can reduce risk of disease complications; delay in diagnosis and global shortages of DAT make this difficult

\section{Learning points}

- Severe diphtheria may occur in partially immunised children in low incidence countries.

- Neurological involvement may be insidious and diagnosis may be delayed in countries with little experience.

- Diptheria associated coronary artery dilatation is not well documented.

- Diptheria anti-toxin can be safely given by subcutaneous infusion; this may be useful to document in future national guidelines.

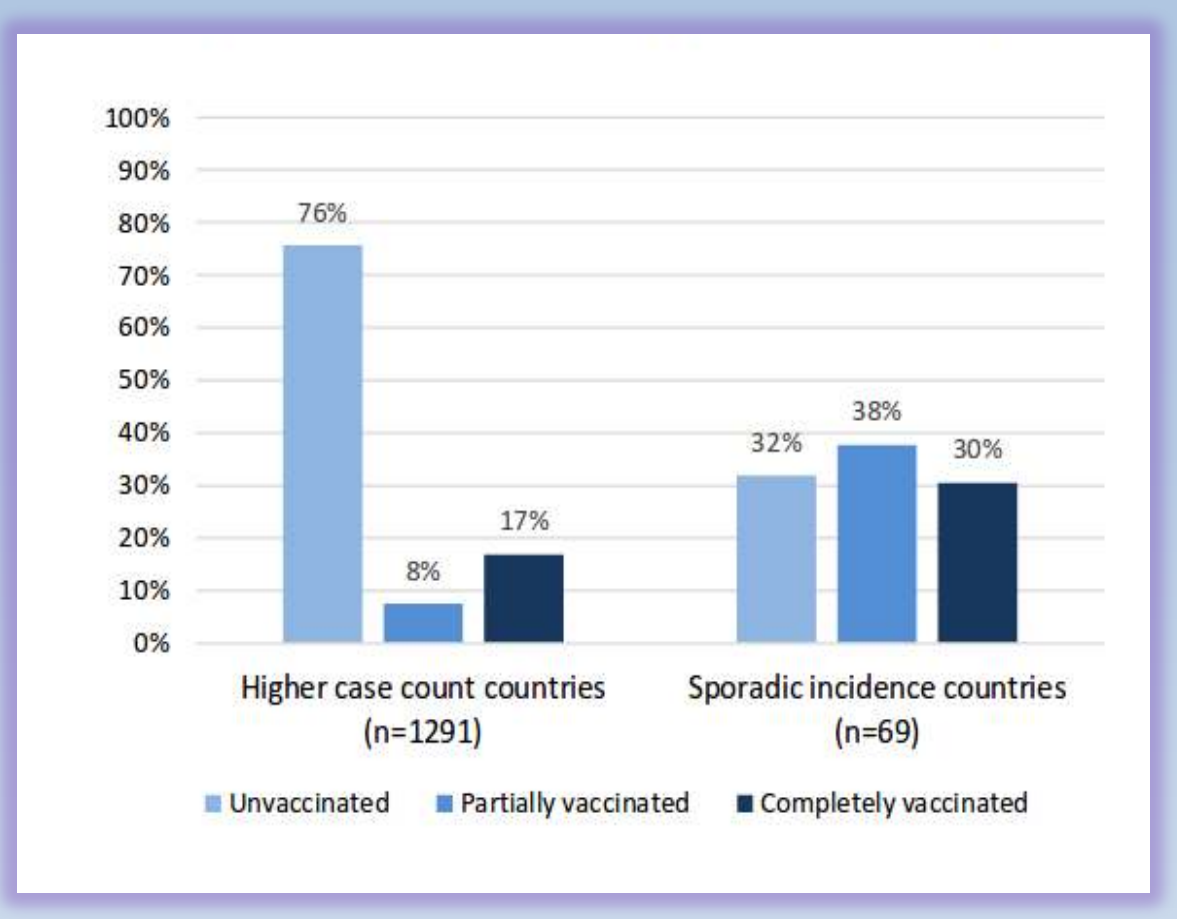

